\title{
Aloetismo em Acromyrmex subterraneus brunneus Forel (Hymenoptera, Formicidae), durante o forrageamento, cultivo do jardim de fungo e devolução dos materiais forrageados
}

\author{
Luiz C. Forti ${ }^{1,2}$, Roberto S. Camargo ${ }^{1}$, Carlos Alberto O. de Matos ${ }^{1}$, Ana Paula P. de Andrade ${ }^{1}$ \\ \& Juliane F. Lopes ${ }^{1}$
}

\begin{abstract}
${ }^{1}$ Laboratório de Insetos Sociais-Praga, Departamento de Produção Vegetal, Faculdade de Ciências Agronômicas, Universidade Estadual Paulista. Caixa Postal 237, 18603-970 Botucatu-SP, Brasil. Endereço eletrônico: luizforti@fca.unesp.br ${ }^{2}$ Pesquisador do CNPq.
\end{abstract}

\begin{abstract}
Alloethism in Acromyrmex subterraneus brunneus Forel (Hymenoptera, Formicidae), during foraging, fungusgarden cultivation and devolution of foraged materials. Leaf cutting-ants present a sophisticated division of labor during foraging, fungus-garden cultivation and devolution of foraged materials. Material with different degrees of moisture and hardness (floral sponge, polystyrene, plastic and clay) were offered to two Acromyrmex subterraneus brunneus Forel, 1911 laboratory colonies. The different size categories of workers were observed during the execution of 14 subtasks. Relative probability performance and alloethic curves were used to verify behavioral flexibility and behavioral stereotyped patterns in the workers. The behavioral patterns could establish roles within probable subcastes.
\end{abstract}

KEYwords. Acromyrmex subterraneus brunneus; alloethism; foraging; leaf-cutting ants.

Resumo. As formigas cortadeiras apresentam uma sofisticada divisão de trabalho durante o forrageamento, cultivo do jardim de fungo e devolução dos materiais forrageados. Materiais com diferentes graus de hidratação e dureza (esponja floral, isopor, plástico e argila) foram oferecidos a duas colônias de laboratório de Acromyrmex subterraneus brunneus Forel, 1911. As diferentes categorias de tamanho de operárias foram observadas durante a execução de 14 subtarefas. Probabilidade relativa de desempenho e as curvas aloéticas foram usadas para verificar a flexibilidade comportamental e os padrões comportamentais estereotipados das operárias. Os padrões comportamentais possibilitaram estabelecer papeis dentro de prováveis subcastas.

Palavras-Chave. Acromyrmex subterraneus brunneus; aloetismo; formigas cortadeiras; forrageamento.

A formação de uma sociedade ocorre quando indivíduos de uma mesma espécie se organizam de modo cooperativo, proporcionando a sustentabilidade e perpetuação da espécie. De acordo com WILSON (1975), quando a sociedade torna-se mais eficiente, maior e geometricamente mais estruturada, seus membros tornam-se especializados dentro de papéis e castas, e seu relacionamento define-se através da comunicação. Tal comunicação permitiu aos animais dividirem o trabalho na sociedade.

Assim, os animais exibiram variações individuais no comportamento, um fenômeno conhecido como polietismo (SudD 1967). O polietismo pode ser etário, quando os indivíduos exibem diferentes comportamentos durante a vida, e por casta, quando apresentam diferenças morfológicas e fisiológicas (HöLLDOBLER \& WILSON 1990).

O polietismo possibilitou a diferenciação dos papéis na sociedade, através da especialização dos indivíduos, promovendo uma mistura otimizada de especialistas que desempenham mais eficientemente o trabalho, do que grupos iguais constituídos de generalistas (WILSON 1968).

Um exemplo notório são as polimórficas formigas cortadeiras (Atta Fabricius, 1804 e Acromyrmex Mayr, 1865), nas quais as maiores operárias são especializadas para a defesa da colônia, operárias médias forrageiam as espécies vegetais e as operárias menores são especializadas para trabalharem dentro do jardim de fungo (WILSON 1980).

O aloetismo se intensifica quando as operárias estão envolvidas no forrageamento e processamento vegetal para a obtenção e geração de recursos alimentares. Tal processo envolve uma série de tarefas específicas realizadas em função do tamanho do corpo das operárias (WILSON 1980, 1983).

Em Atta sexdens rubropilosa Forel, 1908, após o forrageamento, as operárias generalistas (cápsula cefálica entre 1,3 a 1,6 mm) lambem e repicam as folhas em pedaços de 1 a 2 $\mathrm{mm}$ de diâmetro. Em seguida, operárias menores (cápsula cefálica entre 0,8 a 1,2 mm) mastigam ao longo das bordas até a redução das folhas em uma polpa úmida, e inserem tais fragmentos no jardim de fungo (ANDRADE et al. 2002). Finalmente, tufos de hifas são transplantados na superfície dos fragmentos (WEBER 1956).

Para determinar o aloetismo existente em Acromyrmex subterraneus brunneus Forel, 1911 foi realizado um estudo etológico em colônias de laboratório durante o forrageamento, cultivo do jardim de fungo e devolução dos materiais forrageados. 


\section{MATERIALE MÉTODOS}

Este estudo foi realizado em duas colônias de A. s. brunneus, coletadas na cidade de Piracicaba, São Paulo, transportadas e mantidas no Laboratório de Insetos SociaisPraga da Faculdade de Ciências Agronômicas, Universidade Estadual Paulista, Botucatu, São Paulo.

As colônias foram acondicionadas individualmente em recipientes plásticos, nos quais havia uma camada de $1 \mathrm{~cm}$ de gesso, com a finalidade de manter a umidade da cultura de fungo. A temperatura ambiente foi mantida, aproximadamente, a $24^{\circ} \mathrm{C}$, com uma umidade relativa de $70 \%$.

Para o acompanhamento das tarefas realizadas durante o cultivo do fungo simbionte pelas operárias, uma parcela representativa de cada colônia foi transferida para caixas de vidro (comprimento: $20 \mathrm{~cm}$; largura: $10 \mathrm{~cm}$ e altura: $5 \mathrm{~cm}$ ), com $1 \mathrm{~cm}$ de gesso no fundo, interligadas por uma arena de forragem e câmara de lixo em extremidades opostas. As parcelas continham uma população total de 858 e 1025 indivíduos.

As operárias foram separadas por classes de tamanho (muito pequena $0,7-0,8 \mathrm{~mm}$; pequena $0,9-1,1 \mathrm{~mm}$; média 1,2-1,6 $\mathrm{mm}$ e grande 1,7-2,0 $\mathrm{mm}$ ), através de medidas da cápsula cefálica. Posteriormente, realizou-se a marcação do pronoto de todos os indivíduos de cada classe de tamanho, assim diferenciadas por cores.

Para realizar o estudo de polietismo, utilizou-se inertes para simular substratos que continham uma alta quantidade de água e baixa resistência física ao corte (esponja floral), e o contrário, ou seja, substratos que contêm uma baixíssima quantidade de água e altíssima resistência física (plástico), e intermediários como a argila e isopor com baixa resistência física, mas com variação no grau de hidratação. Os inertes foram padronizados em formatos esféricos de aproximadamente $2 \mathrm{~mm}$ de diâmetro, com intuito de anular a seletividade à forma do substrato. Tais inertes foram imersos em uma solução com água e corante alimentar a 0,2\% (CASSIL \& TSCHINKEL 1995) e, em seguida, adicionou-se polpa cítrica seca e moída, para aumentar a atratividade dos mesmos.

As observações foram realizadas sob estereomicroscópio, possibilitando uma visualização detalhada dos atos comportamentais. Nas colônias, foram oferecidos seqüencialmente todos os inertes, com 5 observações de 6 horas para cada tipo, totalizando 20 observações/colônia.

Neste trabalho, adotou-se a terminologia de tarefa e subtarefa. Segundo ANDERSOn et al. (2001), a tarefa é um item da divisão de trabalho, e a subtarefa um conjunto de comportamentos requeridos para completar uma tarefa. As subtarefas registradas foram: 1) carregar inerte para dentro da colônia; 2) transportar fragmento inerte para a superfície do fungo; 3) lamber superfície do inerte; 4) segurar inerte sobre o jardim de fungo, enquanto outras operárias executam outras atividades; 5) repicar inerte, com significativa redução de tamanho (fragmento); 6) mastigar fragmento após estar repicado; 7) incorporar fragmentos do inerte na região apical do jardim de fungo; 8) lamber superfície dos fragmentos incorporados, deixando-os mais úmidos; 9) depositar tufos de

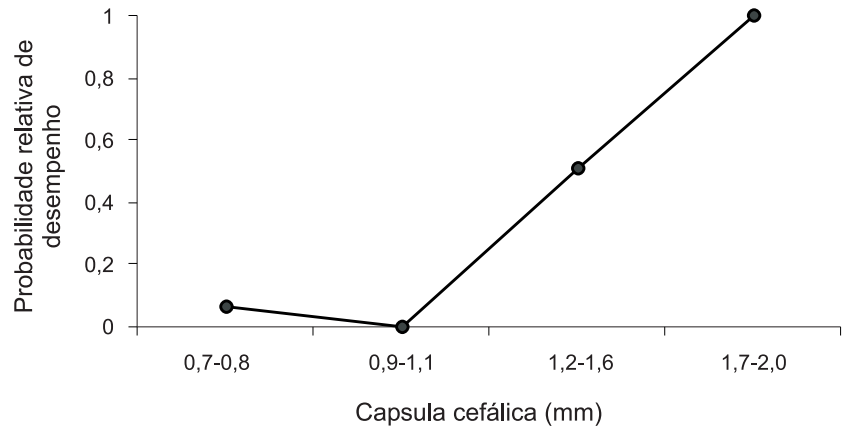

Fig. 1. Probabilidade relativa de desempenho da tarefa de forrageamento em duas colônias de Acromyrmex subterraneus brunneus, em laboratório, Botucatu, SP, 2002.

hifas nos fragmentos incorporados; 10) depositar fluido fecal; 11) devolver fragmentos próximo ao lixo; 12) devolver fragmentos próximo à entrada da arena de forrageamento; 13) transportar fragmento para o lixo; 14) transportar fragmento para a arena de forrageamento.

Estas subtarefas foram distribuídas dentro de três tarefas: forrageamento (1), cultivo do jardim de fungo (2 a 10) e devolução dos materiais forrageados (11 a 14).

A probabilidade relativa de desempenho foi calculada através da probabilidade de ocorrência das freqüências observadas associadas às medidas observadas dos resíduos padronizados em tabela de contingência (PEREIRA 1999). Em cada subtarefa, a maior probabilidade foi considerada como 1 , $\mathrm{e}$ as demais entre $0 \mathrm{e} 1$.

Neste trabalho, os dados de polietismo foram convertidos em probabilidade relativa de desempenho, visando à obtenção das curvas aloéticas (Figs. 1-3). Estas curvas permitem uma fácil interpretação das diferenças comportamentais exibidas pelas diferentes classes de tamanho.

\section{RESULTADOSEDISCUSSÃO}

O aloetismo é um fenômeno comparável das respostas comportamentais, no qual a probabilidade de desempenho de uma tarefa especifica está em função do tamanho do corpo da operária (WILSON 1978; 1980). Tal fenômeno pode ser utilizado como uma relação quantitativa para caracterizar o polietismo.

Dentre as tarefas, o forrageamento e o cultivo do jardim de fungo apresentaram uma forte correlação aloética, devido à mudança gradual nas habilidades com a redução corporal das operárias (Figs. 1 e 2). Observou-se uma maior probabilidade de desempenho para as operárias maiores na subtarefa de carregar inerte para dentro da colônia (Fig. 1). O forrageamento não foi completamente estudado neste trabalho, pois durante a busca de vegetais, as operárias podem executar até 15 atos comportamentais (FORTI \& MARTINS 1987).

Por outro lado, a alternância das curvas aloéticas demonstrou um baixo grau de especialização das operárias para a devolução dos materiais forrageados (Fig. 3), ou seja, operárias de diferentes tamanhos podem realizar quaisquer 

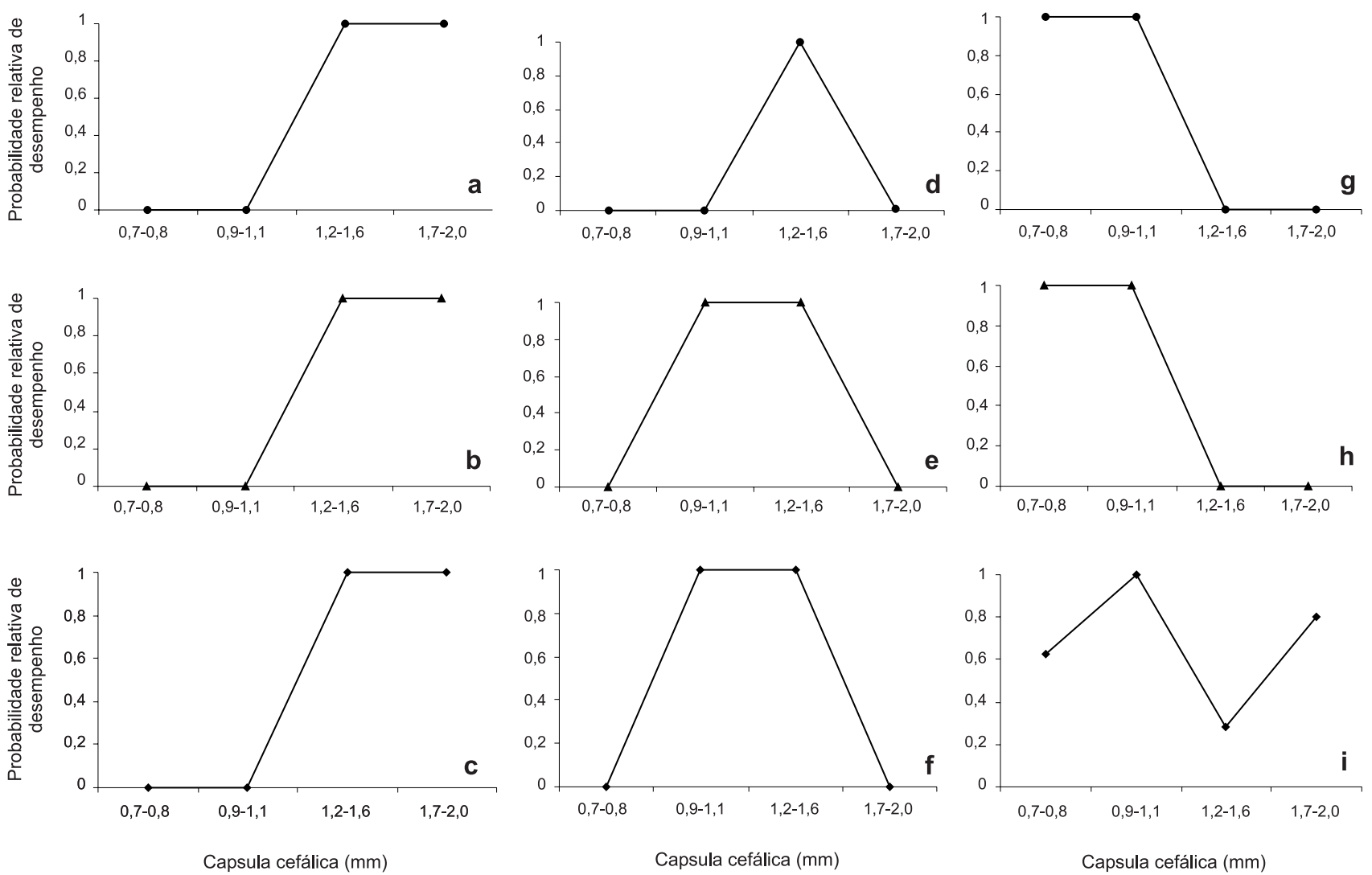

Fig. 2. Probabilidade relativa de desempenho da tarefa do cultivo do jardim de fungo, composta pelas subtarefas: a, transportar fragmento inerte para a superfície do fungo; b, lamber superfície do inerte; c, segurar inerte sobre o jardim de fungo, enquanto outras executam outros atos comportamentais; d, repicar inerte, com significativa redução de tamanho; e, mastigar fragmento após estar repicado; f, incorporar fragmentos do inerte na região apical do jardim de fungo; g, lamber superfície dos fragmentos incorporados, deixando-os mais úmidos; h, depositar tufos de hifas nos fragmentos incorporados; i, depositar fluido fecal; em duas colônias de Acromyrmex subterraneus brunneus, em laboratório, Botucatu, SP, 2002.

destas subtarefas. Estas curvas ilustram a flexibilidade comportamental das operárias, característica essencial para o bom desempenho da colônia.

Segundo Oster \& Wilson (1978), a flexibilidade comportamental e a capacidade de cooperação podem aumentar o repertório de tarefas de um indivíduo. Este fato está correlacionado com a ausência ou a presença de castas físicas. Esses autores demonstraram matematicamente que medidas alométricas estão inversamente correlacionadas com a flexibilidade comportamental. As espécies monomórficas apresentam uma maior probabilidade de desempenho de tarefas por castas, possibilitando uma alta flexibilidade comportamental. Por outro lado, as espécies polimórficas, como as formigas cortadeiras, apresentam uma menor flexibilidade comportamental devido à baixa probabilidade de desempenho de tarefas pelas castas.

Os papéis na sociedade são padrões de comportamento exibidos por determinados indivíduos. Este conjunto de indivíduos são altamente especializados e restritos a um ou mais papéis (WILSON 1985).

Em formigas cortadeiras, os padrões comportamentais ou papéis aparecem repetitivamente em curvas aloéticas no cultivo do jardim de fungo. Para A. s. brunneus, sugere-se a ocorrência de quatro subcastas estabelecidas a partir do agrupamento de papéis das subtarefas (Fig. 4).

A primeira casta é constituída de operárias com cápsula cefálica entre 1,7 a 2,0 mm (operária grande), responsáveis pelas subtarefas a, b e c (Fig. 4). Estas subtarefas estão relacionadas com o início do processamento dos materiais forrageados e exigem menores precisão e minúcia para sua execução.

As operárias médias (cápsula cefálica entre 1,2 a 1,6 mm) foram denominadas como uma segunda subcasta, pois se destacam por executarem, sozinhas, a subtarefa de repicar inerte com significativa redução de tamanho (subtarefa d, Fig. 4) além das subtarefas a, b, c, e, f (Fig. 4). Assim, as operárias médias não podem ser agrupadas com as operárias grandes, devido à responsabilidade de executar este papel.

As operárias pequenas (cápsula cefálica entre 0,9 a 1,1 $\mathrm{mm}$ ) contribuem com as subtarefas e, f (Fig.4), compartilhadas com as operárias médias. As subtarefas $\mathrm{g}, \mathrm{h}$, relacionadas com o final do processo de incorporação de materiais, são realizadas pelas operárias pequenas e muito pequenas (cápsula cefálica entre 0,7 a $0,8 \mathrm{~mm}$ ). A impossibilidade do agrupamento destas 

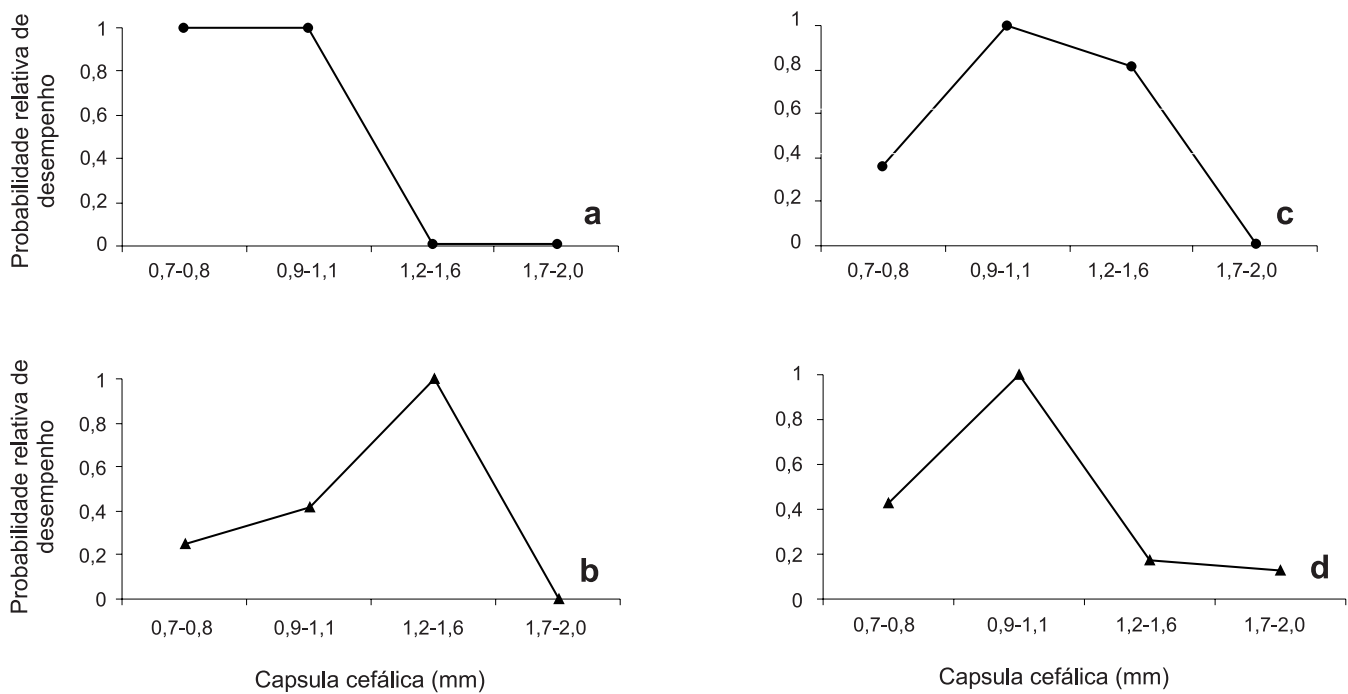

Fig. 3. Probabilidade relativa de desempenho da tarefa de devolução dos materiais forrageados compostas pelas subtarefas: a, devolver fragmentos próximo ao lixo; b, devolver fragmentos próximo à entrada da arena de forragem; c, transportar fragmento para o lixo; d, transportar fragmento para a arena de forragem; em duas colônias de Acromyrmex subterraneus brunneus, em laboratório, Botucatu, SP, 2002.

classes de tamanhos se deve ao fato que as operárias muito pequenas não realizam as subtarefas e, f(Fig. 4), possibilitando a determinação de mais duas castas.

Embora levantada a hipótese da existência de quatro subcastas, um estudo comportamental deve ser realizado enfocando todas as tarefas ou subtarefas realizadas pelas operárias e rainha de A. s. brunneus. Em Atta texana (Buckley, 1860), o polimorfismo e o elevado número de papéis sociais possibilitam a divisão de trabalho dentro de 37 tarefas distintas (Fowler 1983). Talvez, o número de tarefas sociais de $A$. $s$. brunneus seja similar ao encontrado para A. texana.

Dentre os atos comportamentais relatados para formigas, apenas quatro podem ser classificados como um comportamento individual, sem função social (OSTER \& WILSON 1978). Os exemplos são a alimentação, auto-limpeza, toque antenal e excreção.

Em formigas cortadeiras, a urina é denominada de fluido fecal (MARTIN 1970) e é utilizado para o cultivo do jardim de fungo pois contem proteases originárias do fungo, vitais para o seu crescimento em um novo substrato (BOYD \& MARTIN 1975).

Como a deposição do fluido fecal é realizada por uma única operária, não ocorre interação inter- e intracasta. Este comportamento individual difere dos comportamentos de fundo social nas curvas aloéticas, porém sua probabilidade relativa de desempenho é maior que 0 para todas as operárias, enquanto que nos comportamentos sociais a probabilidade varia de $0 \mathrm{a}$ 1 (Fig. 2). Tal comportamento individual pode ser considerado uma adaptação favorável ao cultivo do jardim de fungo, adquirindo assim, uma função social na colônia.

Assim, o estudo comportamental com curvas aloéticas permite relacionar o polimorfismo com o polietismo entre os indivíduos, com o estabelecimento de papéis dentro da organização social das colônias.

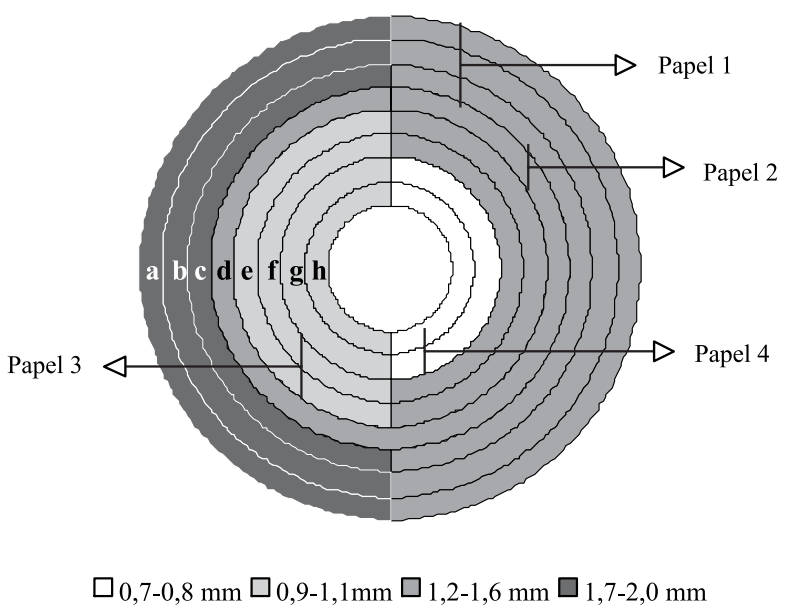

Fig. 4. Etograma com papéis e classes de tamanho precisamente definidos. Cada conjunto de atos comportamentais (Subtarefas) está representado pela probabilidade relativa de desempenho, em cada classe de tamanho. A linha mediana vertical que separa as subtarefas, indica que os papéis são divididos por mais de uma classe de tamanho. Subtarefas: a, transportar fragmento inerte para a superfície do fungo; b, lamber superfície do inerte; c, segurar inerte sobre o jardim de fungo, enquanto outras executam outros atos comportamentais; d, repicar inerte, com significativa redução de tamanho (fragmento); e, mastigar fragmento após estar repicado; f, incorporar fragmentos do inerte na região apical do jardim de fungo; g, lamber superfície dos fragmentos incorporados, deixando-os mais úmidos; $\mathbf{h}$, depositar tufos de hifas nos fragmentos incorporados.

Agradecimentos. À Fundação de Amparo à Pesquisa do Estado de São Paulo (FAPESP) pelo auxílio à pesquisa concedido ao primeiro autor e à bolsa concedida ao segundo autor (Processos: 02/02011-5 e 01/11895-1). 


\section{REFERÊNCIAS}

Anderson, C.; N. R. Franks \& D. W. McShea. 2001. The complexity and hierarchical structure of tasks in insect societies. Animal Behaviour 62: 643-651.

Andrade, A. P. P.; L. C. Forti; A. A. Moreira; M. A. C. Bonretto; V. M. Ramos \& C. A. O. Matos. 2002. Behavior of Atta sexdens rubropilosa (Hymenoptera: Formicidae) workers during the preparation of the leaf substrate for symbiont fungus culture. Sociobiology 40(2): 293-306.

Boyd, N. D. \& M. M. Martin. 1975. Faecal proteinases of the fungusgrowing ant Atta texana: their fungal origin and ecological significance. Journal of Insect Physiology 21: 1815-1820.

Cassill, D. L. \& W. R. Tschinkel. 1995. Allocation of liquid food to larvae via trophallaxis in colonies of the fire ant, Solenopsis invicta. Animal Behaviour 50: 801-813.

Forti, L. C. \& F. S. D. Martins. 1987. Atos comportamentais apresentados pelas operárias forrageiras de Atta sexdens rubropilosa Forel, 1908 (Hymenoptera, Formicidae) durante a coleta de folhas, p. 297-298. In: Encontro Anual de Etologia 5, Anais de Etologia. Jaboticabal, Universidade Estadual Paulista.

Fowler, H. G. 1983. Alloethism in a leaf-cutting ant: laboratory studies on Atta texana (Hymenoptera: Formicidae: Attini). Zoologische Jahrbucher-Abteilung fur Allgemeine Zoologie und Physiologie der Tiere 87(4): 529-538.

Hölldobler, B. \& E. O. Wilson. 1990. The ants. Cambridge, Harvard University Press, $732 \mathrm{p}$.
Martin, M. M. 1970. The biochemical basis of the fungus Attini ant symbiosis. Science 169: 1620.

Oster, G. F. \& E. O. Wilson. 1978. Caste and Ecology in the Social Insects. Princeton, Princeton University Press, 352 p.

Pereira, J. C. R. 1999. Análise de dados qualitativos: estratégias metodológicas para as ciências da saúde, humanas e sociais. São Paulo, EDUSP, 157 p.

Sudd, J. H. 1967. An introduction to the behaviour of ants. London, Edward Arnold Ltda, 200 p.

Weber, N. A. 1956. Treatment of substrate by fungus-growing ants. Anatomical Record 125(3): 604-605.

Wilson, E. O. 1968. The ergonomics of caste in the social insects. American Naturalist 102: 41-66.

Wilson, E. O. 1975. Sociobiology. The new synthesis. Cambridge, Harvard University Press, 697 p.

Wilson, E. O. 1978. Division of labor in fire ants based on physical castes (Hymenoptera: Formicidae: Solenopsis). Journal of the Kansas Entomological Society 51(4): 615-636.

Wilson, E. O. 1980. Caste and division of labor in leaf-cutter ants (Hymenoptera, Formicidae: Atta). I: The overall pattern in A. sexdens. Behavioral Ecology and Sociobiology 7: 143-156.

Wilson, E. O. 1983. Caste and division of labor in leaf-cutter ants (Hymenoptera, Formicidae: Atta). IV: Colony ontogeny of $A$. cephalotes. Behavioral Ecology and Sociobiology 14: 55-60.

Wilson, E. O. 1985. The sociogenesis of insect colonies. Science 228 (4707): 1489-1495. 